\title{
FORTBILDUNG
}

\section{Wie kann man alten Ärzten neue Tricks beibringen?}

"Alte Hasen" unter den Ärzten werden ihre langjährig geübte Routine nicht gleich über Bord werfen, wenn eine neue Leitlinie oder Therapieempfehlung dies nahelegt. Diese Kollegen sind weder fortbildungsunfähig noch -unwillig. Dass neue Erkenntnisse oft lange brauchen, bis sie in den Praxen Einzug halten, liegt an der Art, in der sie propagiert werden, schreibt J. A. Arrighi, Kardiologe am Rhode Island Hospital in einem Editorial der Zeitschrift "Circulation".
Die üblichen Konferenzen mit einem oder mehreren Vortragenden und einer passiven Zuhörerschaft sind nicht der optimale Weg, um neue Erkenntnisse zu verbreiten. Interaktives Lernen mittels Falldiskussionen, Rollenspiel und multimedialen Präsentationen wären wesentlich effektiver. Auch ältere Ärzte könnten neue Tricks lernen, meint Arrighi. Man müsse sich nur mehr Mühe geben, sie ihnen beizubringen.

Circulation, im Druck

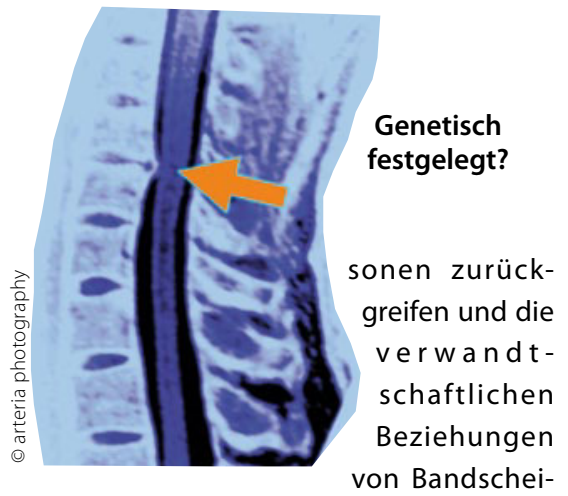

benpatienten untersuchen. Selbst wenn ein relativ entfernter Verwandter an den Bandscheiben erkrankt ist, hat man demnach selbst ein signifikant erhöhtes Risiko, damit ebenfalls Probleme zu bekommen.

The Journal of Bone and Joint Surgery, 2011; 93(3): 225 DOI: 10.2106/JBJS.J.00276

\section{TONSILLEKTOMIE}

\section{Macht Mandel-Op. Kinder dick?}

Dass es nach einer Mandeloperation häufig zu einer Gewichtszunahme kommt, zeigte die Auswertung von neun Studien, in denen das Gewicht vor und ein Jahr nach einer Adenotonsillektomie bei insgesamt 795 Kindern dokumentiert wurde. Ein möglicher Grund könnte sein, dass die Kids beim Essen endlich keine Schmerzen mehr haben. Was bei eher untergewichtigen Kin-

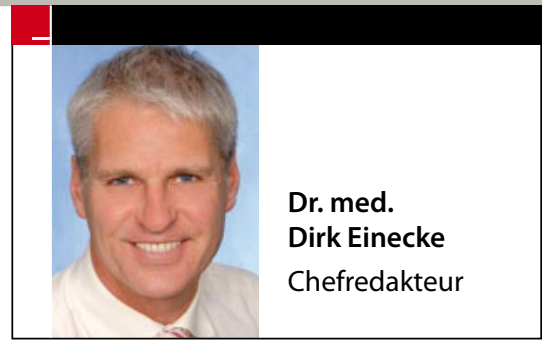

KHK-PRÄVENTION MIT TEE

\section{Grün, nicht schwarz}

Wer sein kardiovaskuläres Risiko senken will, sollte bevorzugt grünen Tee genießen. In einer Metaanalyse von 18 Studien, die den Zusammenhang von Tee und KHK-Endpunkten untersucht hatten, stellten chinesische Wissenschaftler fest, dass Schwarztee das KHK-Risiko nicht wesentlich beeinflusst. Wurde die höchste getrunkene Teemenge der niedrigsten gegenübergestellt, ergab sich ein relatives Risiko von 0,92. Der Wert für Grüntee lag bei 0,72, womit das Signifikanzniveau erreicht war. Pro Tasse Grüntee mehr am Tag sank das KHK-Risiko um relative $10 \%$. Der Effekt sei biologisch plausibel, so Studienleiter Wang: Die in Grüntee enthaltenen Katechine hemmen u. a. Oxidation, Gefäßinflammation, Atherogenese und Thrombogenese. Schwarztee enthält wesentlich weniger dieser protektiven Substanzen.

Am J Clin Nutr 2011; DOI: 10.3945/ajcn.110.005363.

www.SpringerMedizin.de

Top-Herz-Themen

Nutzen Sie die Fülle des Themenangebots auf SpringerMedizin.de! Die jüngsten LeseHits aus dem Fach Kardiologie: _ Kaffee, Alkohol, Fisch - Vorhofflimmern durch Ernährung (325284)

- Blutdrucksenkung: HCT - als FirstLine-Therapie ein Schwächling (328740)

_ Arrhythmien - notwendige Kontrolluntersuchungen bei Therapie mit Dronedaron (328486)

Die o.g. Artikel finden Sie, indem Sie den Titel oder die (in Klammern gesetz te) ID-Nummer in die Suche eingeben.
Otolaryngology - Head and Neck Surgery, 2011 (online dern wünschenswert ist, sollte man aber Ärzte sollten den Eltern von übergewichtigen Kindern deshalb raten, nach der Op. auf eine ausgewogene Ernährung und einen gesunden Lebensstil zu achten. \section{first); DOl: 10.1177/0194599810392328}

